\title{
Painful periods in adolescents
}

\author{
Olga Kciuk MD MSc, Sari Kives MD
}

Cite as: CMAJ 2021 April 19;193:E560. doi: 10.1503/cmaj.201972

CMAJ Podcasts: author interview at www.cmaj.ca/lookup/doi/10.1503/cmaj.201972/tab-related-content

1 品

Dysmenorrhea, defined as pain with menstruation, affects $50 \%-90 \%$ of menstruating adolescents and is the leading cause of recurrent school absenteeism in this population

One-third of menstruating adolescents miss school or sports because of dysmenorrhea. ${ }^{1}$ To minimize impact on academic and social development, physicians should proactively counsel patients on options for managing period pain. ${ }^{1-3}$ First-line treatment can be started before a specific diagnosis is made. ${ }^{2}$

2 Up to $90 \%$ of dysmenorrhea in adolescents occurs in the absence of pelvic pathology (primary dysmenorrhea) and is mediated by excess prostaglandin production Features suggestive of secondary dysmenorrhea (Box 1) should prompt pelvic ultrasonography and may warrant referral to a gynecologist. 2,3

First-line treatment is short-term use of prophylactic nonsteroidal anti-inflammatory drugs (NSAIDs)

Naproxen, ibuprofen and other NSAIDs are equally effective, with a number needed to treat (NNT) of 3 to achieve pain relief in people with primary dysmenorrhea. ${ }^{4}$ Full-strength doses should be taken with food, on a regular schedule with no skipped administrations starting 1-2 days before the onset of menses (if predictable) or at the first sign of bleeding or pain, and continued for the first 2-3 days of bleeding. 2,3

Combined or progestin-only hormonal medications can be added as adjunct therapy

Combined oral contraceptives have an NNT of 5 for treating primary dysmenorrhea. ${ }^{5}$ Combined oral contraceptives with doses of ethinylestradiol above $30 \mu \mathrm{g}$ should be chosen for adolescents, for maintenance of bone health. ${ }^{6}$ Continuous dosing provides more effective relief than standard cyclic use. ${ }^{2,3}$ Levonorgestrelcontaining intrauterine systems and the etonogestrel implant are also safe and effective first-line options. ${ }^{2,3,7}$

Endometriosis is the most common cause of secondary dysmenorrhea in adolescents

Endometriosis is found in as many as $70 \%$ of adolescents who undergo laparoscopy for dysmenorrhea refractory to treatment with NSAIDs and hormonal therapy. ${ }^{3}$ If dysmenorrhea persists beyond 3 months despite adequate first-line treatment, referral to a gynecologist is warranted.
Box 1: Features suggestive of secondary dysmenorrhea ${ }^{2,3}$

- Onset immediately with menarche

- Progressively worsening dysmenorrhea

- Abnormal bleeding (including irregular bleeding) with pain

- Family or personal history of renal or other congenital anomalies (including spine, cardiac, or gastrointestinal)

- Midcycle or acyclic pain

- Dyspareunia

- Family history of endometriosis

\section{References}

1. Armour M, Ferfolja T, Curry C, et al. The prevalence and educational impact of pelvic and menstrual pain in Australia: a national online survey of 4202 young women aged 13-25 years. J Pediatr Adolesc Gynecol 2020;33:511-8.

2. Burnett M, Lemyre M. No. 345-primary dysmenorrhea consensus guideline. J Obstet Gynaecol Can 2017;39:585-95.

3. American College of Obstetricians and Gynecologists. Dysmenorrhea and endometriosis in the adolescent. ACOG Committee opinion no. 760. Obstet Gynecol 2018;132:e249-58.

4. Marjoribanks J, Ayeleke RO, Farquhar C, et al. Nonsteroidal antiinflammatory drugs for dysmenorrhoea. Cochrane Database Syst Rev 2015;(7):CD001751.

5. Wong $\mathrm{CL}$, Farquhar $\mathrm{C}$, Roberts $\mathrm{H}$, et al. Oral contraceptive pill for primary dysmenorrhea. Cochrane Database Syst Rev 2009;(4):CD002120.

6. Golden NH. Bones and birth control in adolescent girls. J Pediatr Adolesc Gynecol 2020;33:249-54.

7. Bayer LL, Hillard PJA. Use of levonorgestrel intrauterine system for medical indications in adolescents. J Adolesc Health 2013;52:S54-8.

\section{Competing interests: None declared.}

This article has been peer reviewed.

Affiliations: Department of Obstetrics and Gynaecology, University of Toronto (Kciuk, Kives); The Hospital for Sick Children, Toronto (Kives), Toronto, Ont.

Content licence: This is an Open Access article distributed in accordance with the terms of the Creative Commons Attribution (CC BY-NC-ND 4.0) licence, which permits use, distribution and reproduction in any medium, provided that the original publication is properly cited, the use is noncommercial (i.e., research or educational use), and no modifications or adaptations are made. See: https://creativecommons.org/licenses/by-nc-nd/4.0/

Correspondence to: Olga Kciuk, olga.kciuk@mail.utoronto.ca 